\author{
Marquette University \\ e-Publications@Marquette
}

Electrical and Computer Engineering Faculty Research and Publications

Electrical and Computer Engineering,

Department of

\title{
A New Look at Azimuthal Wave Propagation Constants of an n- Layered Dielectric Coated PEC Cylinder
}

\author{
Jason V. Paul \\ United States Air Force \\ Peter J. Collins \\ United States Air Force Academy \\ Ronald A. Coutu Jr. \\ Marquette University, ronald.coutu@marquette.edu
}

Follow this and additional works at: https://epublications.marquette.edu/electric_fac

Part of the Computer Engineering Commons, and the Electrical and Computer Engineering Commons

\section{Recommended Citation}

Paul, Jason V.; Collins, Peter J.; and Coutu, Ronald A. Jr., "A New Look at Azimuthal Wave Propagation Constants of an n-Layered Dielectric Coated PEC Cylinder" (2013). Electrical and Computer Engineering Faculty Research and Publications. 327.

https://epublications.marquette.edu/electric_fac/327 
Marquette University

\section{e-Publications@Marquette}

\section{Electrical and Computer Engineering Faculty Research and Publications/College of Engineering}

This paper is NOT THE PUBLISHED VERSION; but the author's final, peer-reviewed manuscript. The published version may be accessed by following the link in the citation below.

IEEE Transactions on Components, Packaging and Manufacturing Technology, Vol. 61, No. 5, (January, 2013): 2727-2734. DOI. This article is (C) Institute of Electrical and Electronic Engineers (IEEE) and permission has been granted for this version to appear in ePublications@Marquette. IEEE does not grant permission for this article to be further copied/distributed or hosted elsewhere without the express permission from IEEE.

\section{Contents}

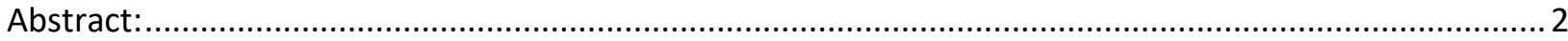

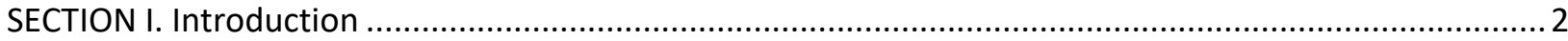

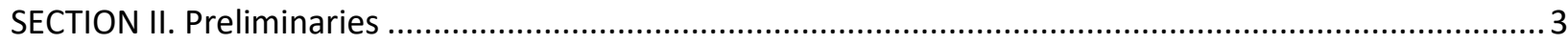

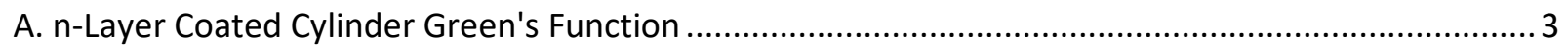

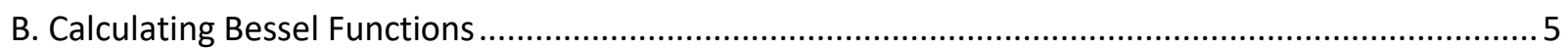

SECTION III. Solving for Azimuthal Wave Propagation Constants ........................................................ 5

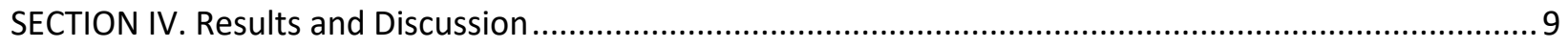

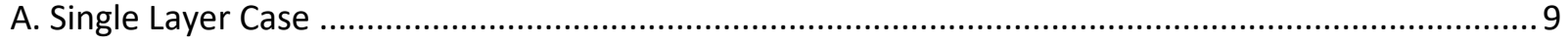

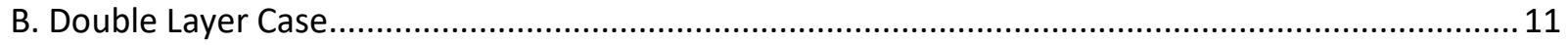

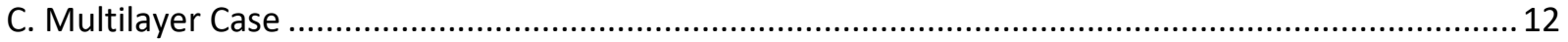

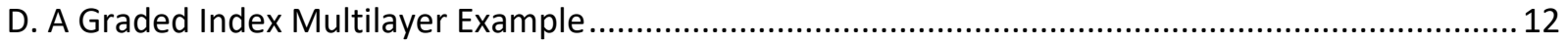

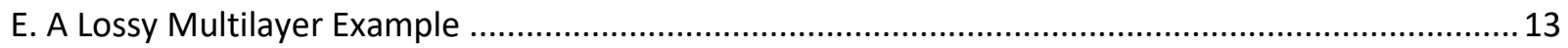

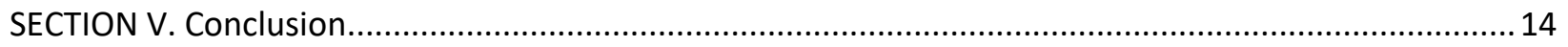

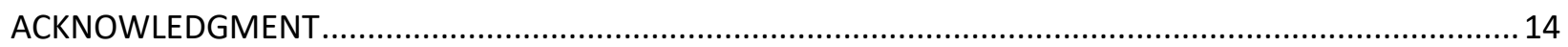

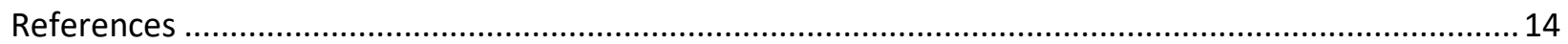




\title{
A New Look at Azimuthal Wave Propagation Constants of an n-Layered Dielectric Coated PEC Cylinder
}

\author{
Jason V. Paul \\ Department of Electrical and Computer Engineering, Air Force Institute of Technology, Wright- \\ Patterson AFB \\ Peter J. Collins \\ Department of Electrical and Computer Engineering, Air Force Institute of Technology, Wright- \\ Patterson AFB
}

Ronald A. Coutu

Department of Electrical and Computer Engineering, Air Force Institute of Technology, WrightPatterson AFB

\section{Abstract:}

A method for determining the azimuthal wave propagation constants supported by an $n$-layered dielectric coated PEC cylinder is presented. The method, based on a Green's function described in , is designed to optimally handle layered cylinders where the number of layers is extremely large as might be encountered in structures designed using transformational optics. The method is also tractable for any stratification profile without the need for individual layer analysis. We implement a recently developed numerical method to calculate Bessel functions of complex order and argument. Our method is verified by comparison with previously published results. We also present new results for a 5-layer case demonstrating self consistency and improved accuracy over published methods. Finally, to illustrate the method's benefits, we present a brief analysis of two multilayer structures; a multilayer variation of the coated cylinder presented in and an example of a 7-layer case that approximates a material parameter gradient.

\section{SECTION I. Introduction}

Waves traveling along a curved surface have been a topic of interest for the past $50+$ years. ${ }^{3,4}$ The study of this topic has many applications including the design of conformal antennas and scattering of cylinders. Specifically, the research has addressed the case of uncoated cylinders, ${ }^{4,5}$ single layer coated cylinders, ${ }^{6-7,8,9,10,11}$ double layer coated cylinders ${ }^{2,12}$ and a multilayered coated cylinder. ${ }^{13}$ The propagation constants of these azimuthal waves can be solved in several ways including the determination of the poles of an associated Green's function.

With the recent research into transformational optics ${ }^{14}$ and metamaterials, new structures are being designed in which the study of azimuthal waves is directly applicable. In 2006, Schurig et al. showed that a cloaked cylinder was theoretically possible. ${ }^{15}$ The literature contains many numerical studies of this 
structure but relatively few analytic studies of the field propagation within the cylindrical layers. Since the cloaked cylinder is a dielectric coated cylinder with a material parameter gradient which guide the fields around the cylinder ideally without reflection, we can leverage past azimuthal wave research to describe the field behavior in this structure. However, to solve for the propagation constants, a method is needed for the case of a n-layered cylinder to approximate the cloak coating material parameter gradient.

In, ${ }^{13}$ a ray-optic based approach implementing asymptotically evaluated Bessel functions was presented including the results from a 2-layered geometry. This method is not suitable because it is a technique applied to a specific stratification profile and not generalizable. This method also becomes intractable as the number of layers gets larger since the analysis quickly becomes unwieldy. This, combined with the asymptotic limitations of the ray-optic approach, drive the requirements for a new method that can be used to analyze the fields within a cloaked cylinder structure where the material parameter gradient is approximated by a large number of layers.

In this article, we present a Green's function approach to solving for the azimuthal propagation constants of an n-layered cylinder which addresses the limitations in current literature. This method takes advantage of the recursive boundary conditions much like the method presented in, ${ }^{16}$ but further refines the expressions into compact, tractable forms where the denominator and numerator can be solved individually for any of the given coefficients. It should be emphasized we are not developing a new Green's function per se. Rather our contribution is the development of new computationally efficient forms for systematically analyzing arbitrary layered structures. The method is designed for coding in that the expressions are generated by the number of layers and not unique to the layer profile. That is, unlike the ray-optic approach of, ${ }^{13}$ we do not have to perform new analysis for every stratification profile. To calculate the possibly complex argument and complex order Bessel functions, we use a recently developed low error numerical method. ${ }^{17}$ Our results are then compared with the previously published results of. . $, 6,12,18$

\section{SECTION II. Preliminaries}

In this section we first present the Green's function and the relevant boundary conditions. Then we cover the method of evaluating complex order and complex argument Bessel functions. A time dependence of $e^{j \omega t}$ is assumed and suppressed.

\section{A. n-Layer Coated Cylinder Green's Function}

The Green's function we use is presented $\mathrm{in}^{1}$ and the full derivation can be seen in. ${ }^{19}$ The relevant geometry is shown in Fig. 1 and it is important to note that this Green's function assumes that the source and observer will always be outside the stratified media.

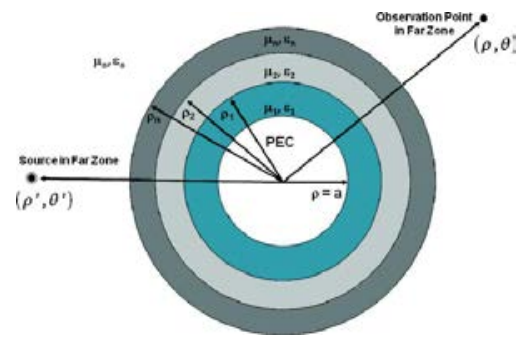

Fig. 1. n-layered Dielectric Coated PEC Cylinder Geometry. ${ }^{1}$ 
The Green's function is given as $\underline{(1)}$

$$
\begin{aligned}
G= & -\frac{j}{4} \sum_{v=0}^{\infty} \frac{\epsilon_{v}}{A_{v}^{n+1}} \cos \left[v\left(\theta-\theta^{\prime}\right)\right] \\
& \times\left[A_{v}^{n+1} J_{v}\left(k_{i} \rho\right)+B_{v}^{n+1} H_{v}^{(2)}\left(k_{i} \rho\right)\right] H_{v}^{(2)}\left(k_{0} \rho^{\prime}\right)(1) \\
A_{v}^{n+1}= & 1 \\
B_{v}^{1}= & -A_{v}^{1} K_{v}
\end{aligned}
$$

where $\epsilon_{v}$ is the Neumann number, $K_{v}=\left(J_{v}^{\prime}\left(k_{1} a\right) / H_{v}^{\prime(2)}\left(k_{1} a\right)\right)$ for TE incidence, $K_{v}=$ $\left(J_{v}\left(k_{1} a\right) / H_{v}^{(2)}\left(k_{1} a\right)\right)$ for TM incidence. There are $n$ layers in this geometry and the superscript of the $A_{v}^{1}, B_{v}^{1}$ variables denotes the associated ith layer. The $A_{v}^{n+1}, B_{v}^{n+1}$ variables correspond with the free space region outside of the layered cylinder and $B_{v}^{n+1}$ is solved through the coefficients $A_{v}^{1}, B_{v}^{1}$ by enforcing continuity boundary conditions of the tangential fields at the layer interfaces

These boundary conditions are

$$
\begin{aligned}
\left.H_{z}\right|_{\rho-} & =\left.H_{z}\right|_{\rho+} \\
\left.\frac{1}{\epsilon^{-}} \frac{\partial}{\partial \rho} H_{z}\right|_{\rho-} & =\left.\frac{1}{\epsilon^{+}} \frac{\partial}{\partial \rho} H_{z}\right|_{\rho+}
\end{aligned}
$$

for the TE case and

$$
\begin{aligned}
\left.E_{z}\right|_{\rho-} & =\left.E_{z}\right|_{\rho+} \\
\left.\frac{1}{\mu^{-}} \frac{\partial}{\partial \rho} E_{z}\right|_{\rho-} & =\left.\frac{1}{\mu^{+}} \frac{\partial}{\partial \rho} E_{z}\right|_{\rho+}
\end{aligned}
$$

for the TM case. ${ }^{20}$

In order to solve for $A_{v}^{i}, B_{v}^{i}$ coefficients, we need to set up the boundary conditions at each interface as seen in (6), where ' denotes the derivative with respect to the argument. This results in a system of equations which can be represented as a matrix equation. ${ }^{19}$ Simply solving for the $A_{v}^{i}, B_{v}^{i}$ coefficients through the matrix is not helpful in this effort since we are interested in the behavior of these constants as a function of the layer constitutive parameters and thicknesses. Specifically, the poles of these coefficient equations contains information on the supported fields. We must algebraically solve the matrix for the denominators of $A_{v}^{i}, B_{v}^{i}$ in a general form so the n-layered case can be solved. This will be discussed in Section III 


$$
\begin{aligned}
& A_{v}^{1}\left[J_{v}\left(k_{1} \rho_{1}\right)-K_{v} H_{v}^{(2)}\left(k_{1} \rho_{1}\right)\right] \\
& =A_{v}^{2} J_{v}\left(k_{2} \rho_{1}\right)+B_{v}^{2} H_{v}^{(2)}\left(k_{2} \rho_{1}\right) \\
& A_{v}^{1} \frac{\kappa_{2}}{k_{1}}\left(J_{v}^{\prime}\left(k_{1} \rho_{1}\right)-K_{v} H_{v}^{\prime(2)}\left(k_{1} \rho_{1}\right)\right) \\
& =\frac{\kappa_{1}}{k_{2}}\left(A_{v}^{2} J_{v}^{\prime}\left(k_{2} \rho_{1}\right)+B_{v}^{2} H_{v}^{\prime(2)}\left(k_{2} \rho_{1}\right)\right) \\
& A_{v}^{2} J_{v}\left(k_{2} \rho_{2}\right)+B_{v}^{2} H_{v}^{(2)}\left(k_{2} \rho_{2}\right) \\
& =A_{v}^{3} J_{v}\left(k_{3} \rho_{2}\right)+B_{v}^{3} H_{v}^{(2)}\left(k_{3} \rho_{2}\right) \\
& \frac{\kappa_{3}}{k_{2}}\left(A_{v}^{2} J_{v}^{\prime}\left(k_{2} \rho_{2}\right)+B_{v}^{2} H_{v}^{\prime(2)}\left(k_{2} \rho_{2}\right)\right) \\
& =\frac{\kappa_{2}}{k_{3}}\left(A_{v}^{3} J_{v}^{\prime}\left(k_{3} \rho_{2}\right)+B_{v}^{3} H_{v}^{\prime(2)}\left(k_{3} \rho_{2}\right)\right) \\
& A_{v}^{n} J_{v}\left(k_{n} \rho_{n}\right)+B_{v}^{n} H_{v}^{(2)}\left(k_{n} \rho_{n}\right) \\
& =J_{v}\left(k_{0} \rho_{n}\right)+B_{v}^{n+1} H_{v}^{(2)}\left(k_{0} \rho_{n}\right) \\
& \frac{1}{k_{n}}\left(A_{v}^{n} J_{v}^{\prime}\left(k_{n} \rho_{n}\right)+B_{v}^{n} H_{v}^{\prime(2)}\left(k_{n} \rho_{n}\right)\right) \\
& =\frac{\kappa_{n}}{k_{0}}\left(J_{v}^{\prime}\left(k_{0} \rho_{n}\right)+B_{v}^{n+1} H_{v}^{\prime(2)}\left(k_{0} \rho_{n}\right)\right) \\
& \kappa=\left\{\begin{array}{l}
\epsilon_{r}=\frac{\epsilon}{\epsilon_{0}} T E \\
\mu_{r}=\frac{\mu}{\mu_{0}} T M
\end{array} .\right.
\end{aligned}
$$

\section{B. Calculating Bessel Functions}

In order to investigate the functional dependence of the $A_{v}^{i}, B_{v}^{i}$ coefficients, we need an efficient method to numerically calculate Bessel functions of complex order and argument. Most efforts in the literature have relied on asymptotic forms, namely the Debye/Watson ${ }^{2,12}$ or Olver $^{2,8,18,21}$ approximations. Accurately calculating the Bessel functions is critical when dealing with a stratified media since any error will be compounded and become greater as the number of layers increases. We chose to use the numerical approach from ${ }^{17}$ since it is valid everywhere and claims an error of less than $6.24 \times 10^{-14}$ in Wronskian tests. In addition, the algorithm is relatively efficient which is critical to our root finding algorithm.

\section{SECTION III. Solving for Azimuthal Wave Propagation Constants}

Surface waves are created along the coated cylinder when the scattered portion of the Green's function encounters a pole and subsequently goes to $\infty .^{22}$ The order at which this pole occurs is the azimuthal propagation constant. The Green's function presented in (1) is made up of two portions, the response due to the incident field and the response due to the scattered field (7)

$$
G_{\text {total }}=G_{\text {incident }}+G_{\text {scattered }} \cdot(7)
$$


The scattered contribution of the Green's function can be written as

$$
\begin{gathered}
G_{\text {scattered }}=-\frac{j}{4} \sum_{v=0}^{\infty} \epsilon_{v} \cos \left[v\left(\theta-\theta^{\prime}\right)\right] \\
\times B_{v}^{n+1} H_{v}^{(2)}\left(k_{i} \rho\right) H_{v}^{(2)}\left(k_{0} \rho^{\prime}\right) .
\end{gathered}
$$

From (8), it is apparent the poles of the scattered portion of the Green's function are contained in $B_{v}^{n+1}$. Since $B_{v}^{n+1}$ can be written in terms of $A_{v}^{1}$, we need to find an expression for $A_{v}^{1}$. Due to the repetitive nature of the boundary conditions in (6), we can get a general expression for $A_{v}^{1}$ that can be used to solve for the propagation constants of any stratification profile.

To solve for $A_{v}^{1}$ in (6), we must work from the boundary conditions of the inner layer to those of the outer layer until we have a system of 2 equations were $A_{v}^{1}$ is a function of $B_{v}^{n+1}$. This system of equations can then be used to solve for an expression for $A_{v}^{1}$ and therefore an expression to solve for the poles.

To simplify this process, we introduce the following variables which are derived from the boundary conditions at the $n$th layer and free space junction

$$
\begin{array}{cc}
D_{v}= & \frac{\pi \rho_{n} k_{n}}{j 2}\left[\frac{\kappa_{n}}{k_{0}} H_{v}^{\prime(2)}\left(k_{0} \rho_{n}\right) H_{v}^{(2)}\left(k_{n} \rho_{n}\right)\right. \\
& \left.-\frac{1}{k_{n}} H_{v}^{\prime(2)}\left(k_{n} \rho_{n}\right) H_{v}^{(2)}\left(k_{0} \rho_{n}\right)\right] \\
E_{v}= & \frac{\pi \rho_{n} k_{n}}{j 2}\left[\frac{\kappa_{n}}{k_{0}} J_{v}^{\prime}\left(k_{0} \rho_{n}\right) H_{v}^{(2)}\left(k_{n} \rho_{n}\right)\right. \\
& \left.-\frac{1}{k_{n}} H_{v}^{\prime(2)}\left(k_{n} \rho_{n}\right) J_{v}\left(k_{0} \rho_{n}\right)\right] \\
F_{v}=\quad \frac{\pi \rho_{n} k_{n}}{j 2}\left[\frac{1}{k_{n}} J_{v}^{\prime}\left(k_{n} \rho_{n}\right) H_{v}^{(2)}\left(k_{0} \rho_{n}\right)\right. \\
\left.\quad-\frac{\kappa_{n}}{k_{0}} H_{v}^{\prime(2)}\left(k_{0} \rho_{n}\right) J_{v}\left(k_{n} \rho_{n}\right)\right] \\
\quad \frac{\pi \rho_{n} k_{n}}{j 2}\left[\frac{1}{k_{n}} J_{v}^{\prime}\left(k_{n} \rho_{n}\right) J_{v}\left(k_{0} \rho_{n}\right)\right. \\
\left.G_{v}=\frac{\kappa_{n}}{k_{0}} J_{v}^{\prime}\left(k_{0} \rho_{n}\right) J_{v}\left(k_{n} \rho_{n}\right)\right]
\end{array}
$$

which allows us to express the boundary conditions of the last layer from $\underline{(6)}$ in the compact form of (10)

$$
\begin{aligned}
& A_{v}^{n}=B_{v}^{n+1} D_{v}+E_{v} \\
& B_{v}^{n}=B_{v}^{n+1} F_{v}+G_{v} .
\end{aligned}
$$

Next, in (11), we define variables which are derived from boundary conditions for the intermediate layers $1<i<n$ where $i$ is the index that refers to the intermediate layer under examination 


$$
\begin{array}{cc}
X_{v}^{i}= & \frac{\pi k_{i} \rho_{i}}{j 2 \kappa_{i+1}}\left[\frac{\kappa_{i}}{k_{i+1}} H_{v}^{\prime(2)}\left(k_{i+1} \rho_{i}\right) H_{v}^{(2)}\left(k_{i} \rho_{i}\right)\right. \\
& \left.-\frac{\kappa_{i+1}}{k_{i}} H_{v}^{(2)}\left(k_{i+1} \rho_{i}\right) H_{v}^{\prime(2)}\left(k_{i} \rho_{i}\right)\right] \\
Y_{v}^{i}= & \frac{\pi k_{i} \rho_{i}}{j 2 \kappa_{i+1}}\left[\frac{\kappa_{i}}{k_{i+1}} H_{v}^{(2)}\left(k_{i} \rho_{i}\right) J_{v}^{\prime}\left(k_{i+1} \rho_{i}\right)\right. \\
& \left.-\frac{\kappa_{i+1}}{k_{i}} J_{v}\left(k_{i+1} \rho_{i}\right) H_{v}^{\prime(2)}\left(k_{i} \rho_{i}\right)\right] \\
R_{v}^{i}=\quad & \frac{\pi k_{i} \rho_{i}}{j 2 \kappa_{i+1}}\left[\frac{\kappa_{i+1}}{k_{i}} J_{v}\left(k_{i+1} \rho_{i}\right) J_{v}^{\prime}\left(k_{i} \rho_{i}\right)\right. \\
S_{v}^{i}= & \left.\quad-\frac{\kappa_{i}}{k_{i+1}} J_{v}^{\prime}\left(k_{i+1} \rho_{i}\right) J_{v}\left(k_{i} \rho_{i}\right)\right] \\
& \quad-\frac{\kappa_{i} \rho_{i}}{k_{i+1}}\left[\frac{\kappa_{i+1}}{k_{i}} H_{v}^{(2)}\left(k_{i+1} \rho_{i}\right) J_{v}^{\prime}\left(k_{i+1} \rho_{i}\right) H_{v}^{\prime(2)}\left(k_{i+1} \rho_{i}\right)\right] .
\end{array}
$$

This allows us to write the general form of the ith intermediate boundary conditions as (12)

$$
\begin{aligned}
& A_{v}^{i}=A_{v}^{i+1} Y_{v}^{i}+B_{v}^{i+1} X_{v}^{i} \\
& B_{v}^{i}=A_{v}^{i+1} R_{v}^{i}+B_{v}^{i+1} S_{v}^{i} .
\end{aligned}
$$

Finally, we repeat the process to get $A 1 v$ from the boundary conditions at the junction between the first and second layers and present the variables in (13)-(15)

$$
\begin{aligned}
z_{n}= & j 2 \kappa_{1} \\
z_{d}= & \pi k_{2} \rho_{1}\left[\kappa_{2} H_{v}^{(2)}\left(k_{2} \rho_{1}\right) Q_{v}\right. \\
& \left.-\frac{\kappa_{1}}{k_{2}} H_{v}^{\prime(2)}\left(k_{2} \rho_{1}\right) P_{v}\right] \\
w_{n}= & j 2 \kappa_{1} \\
w_{d}= & \pi k_{2} \rho_{1}\left[\frac{\kappa_{1}}{k_{2}} J_{v}^{\prime}\left(k_{2} \rho_{1}\right) P_{v}-\kappa_{2} J_{v}\left(k_{2} \rho_{1}\right) Q_{v}\right] \\
P_{v}= & J_{v}\left(k_{1} \rho_{1}\right)-K_{v} H_{v}^{(2)}\left(k_{1} \rho_{1}\right) \\
Q_{v}= & \frac{1}{k_{1}}\left(J_{v}^{\prime}\left(k_{1} \rho_{1}\right)-K_{v} H_{v}^{\prime(2)}\left(k_{1} \rho_{1}\right)\right) \\
Z= & \frac{z_{n}}{z_{d}} \\
W= & \frac{w_{n}}{w_{d}}
\end{aligned}
$$

We can then express a system of equations for $A 1 v$ as $\underline{(16)}$ and $\underline{(17)}$ 


$$
\begin{aligned}
& A_{v}^{1}=A_{v}^{2} Z \\
& A_{v}^{1}=B_{v}^{2} W .
\end{aligned}
$$

It is apparent that while the inner and outer boundary conditions $\underline{(16)}$ and $\underline{(17)}, \underline{(10)}$ will not change for a given stratification profile of 2 or more layers, the intermediate boundary conditions are dependent upon the number of layers.

In an effort to generate expressions for $A_{v}^{2}, B_{v}^{2}$ of $(16)$ and $(17)$ for any given stratification profile, we leverage the predictability of the cascading intermediate boundary conditions. This can best be seen in the tree diagram shown in Fig. 2 which is generated from the intermediate boundary condition $A_{v}^{2}=$ $A_{v}^{3} Y_{v}^{2}+B_{v}^{3} X_{v}^{2}$ and substituting every subsequent intermediate boundary condition of a 5 layer dielectric coated cylinder.

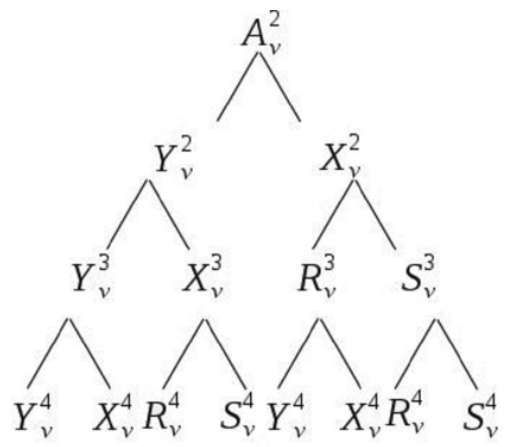

(T) $(M)(T)(M)(T)(M)(T) \quad(M)$

Fig. 2. Substitution tree of a 5 layer dielectric coated cylinder created by solving for $A_{v}^{2}$ with the intermediate boundary conditions.

The variables $T$ and $M$ account for the intermediate boundary conditions and are made of the product of the variables along the path from the bottom to the top of the tree. Likewise, a tree generated from the boundary condition $B_{v}^{2}=A_{v}^{3} R_{v}^{2}+B_{v}^{3} S_{v}^{2}$ will generate the variables $L$ and $M$.

We specify variables, $T_{v}^{n}, M_{v}^{n}, L_{v}^{n}, N_{v}^{n}$, as being made of a summation of $2^{n-2}$ products of $n-2$ variables. For instance, the expressions for the 5-layered cylinder can be generated from Fig. 2 and can be seen in $\underline{(18)}$

$$
\begin{aligned}
T_{v}^{5} & =Y_{v}^{2} Y_{v}^{3} Y_{v}^{4}+Y_{v}^{2} X_{v}^{3} R_{v}^{4}+X_{v}^{2} R_{v}^{3} Y_{v}^{4}+X_{v}^{2} S_{v}^{3} R_{v}^{4} \\
M_{v}^{5} & =Y_{v}^{2} Y_{v}^{3} X_{v}^{4}+Y_{v}^{2} X_{v}^{3} S_{v}^{4}+X_{v}^{2} R_{v}^{3} X_{v}^{4}+X_{v}^{2} S_{v}^{3} S_{v}^{4} \\
L_{v}^{5} & =R_{v}^{2} Y_{v}^{3} Y_{v}^{4}+R_{v}^{2} X_{v}^{3} R_{v}^{4}+S_{v}^{2} R_{v}^{3} Y_{v}^{4}+S_{v}^{2} S_{v}^{3} R_{v}^{4} \\
N_{v}^{5} & =R_{v}^{2} Y_{v}^{3} X_{v}^{4}+R_{v}^{2} X_{v}^{3} S_{v}^{4}+S_{v}^{2} R_{v}^{3} X_{v}^{4}+S_{v}^{2} S_{v}^{3} S_{v}^{4} .
\end{aligned}
$$

We use the superscript and subscript to denote the dependence on the number of layers and order of the Bessel functions respectively. With (18), we can solve the system of boundary condition equations in (10) and (12) to get general expressions of $A_{v}^{2}$ and $B_{v}^{2}$ in terms of $B_{v}^{n+1}$ as seen in (19)

$$
\begin{aligned}
& A_{v}^{2}=\left(B_{v}^{n+1} D_{v}+E_{v}\right) T_{v}^{n}+\left(B_{v}^{n+1} F_{v}+G_{v}\right) M_{v}^{n} \\
& B_{v}^{2}=\left(B_{v}^{n+1} D_{v}+E_{v}\right) L_{v}^{n}+\left(B_{v}^{n+1} F_{v}+G_{v}\right) N_{v}^{n} .
\end{aligned}
$$


Then, substituting the general form of $(19)$ into (16) and (17), we get the system of 2 equations where $A_{v}^{1}$ is in terms of $B_{v}^{n+1}$. By solving this system of equations, we can get general expressions which can identify the poles of the scattering portion of the Green's function.

Due to the form of the boundary conditions, we must split the solution into 3 cases: $n=1, n=2, n>$ 2 . The equation to solve for the poles of the single layer case can be seen in (20),

$$
Q_{v} H_{v}^{(2)}\left(k_{0} \rho_{1}\right)-\frac{\kappa_{1}}{k_{0}} P_{v} H_{v}^{\prime(2)}\left(k_{0} \rho_{1}\right)=0 .(20)
$$

Next, the double layer case can be seen in $\underline{(21)}$

$$
w_{d} D_{v}-z_{d} F_{v}=0(21)
$$

Lastly, the case of $n>2$ is

$$
w_{d}\left(D_{v} T_{v}^{n}+F_{v} M_{v}^{n}\right)-z_{d}\left(D_{v} L_{v}^{n}+F_{v} N_{v}^{n}\right)=0 . \text { (22) }
$$

We use the secant method to solve (20), (21) and (22), first starting with the poles of the uncoated cylinder then refining the initial guesses to ensure we track along the same mode as the thickness of the coating is increased. In this development we begin from the inner layer boundary conditions and work to the outer, but identical results can be obtained by starting at the outer boundary conditions and working towards the inner. It is also important to note that $(20)$ is identical to (3) in. ${ }^{6}$

\section{SECTION IV. Results and Discussion}

In this section we present results of a single layer, double layer and 5-layer case to validate (20), (21) and (22) respectively. The results from ${ }^{6,18}$ are duplicated for the single layer case and the results from ${ }^{2,12}$ are duplicated for the double layer case. To the authors' knowledge, there are no directly comparable published results of the azimuthal propagation constants of a coated cylinder with more than two layers. So to validate a multilayer case we implement a 5-layer structure with the same material parameters and outside diameter of the single layer case.

After validating our method, we conclude with two examples intended to illustrate our method's usefulness. These are both based on multilayer variations of published 2-layer, substrate-superstrate coated cylinders where we use our method to explore the impact of introducing structure variations. In the first example, we provide an example 7-layer structure that implements a lossless material gradient approximation to the 2-layer structure of. ${ }^{12}$ Our second example explores the impact of subdividing the substrate layer of the structure explored in Sun's paper ${ }^{2}$ into high-low dielectric constant layers, along with introducing loss into various layers.

\section{A. Single Layer Case}

In, ${ }^{6}$ Paknys and Wang use a Green's function to determine the azimuthal propagation constants of a single layer coated cylinder for TE and TM incidence. Our results for the TE case can be seen in Fig. 3 and the TM case in Fig. 4 accompanied by the results from. ${ }^{6}$ These graphs show the azimuthal propagation constants for different coating thicknesses. 


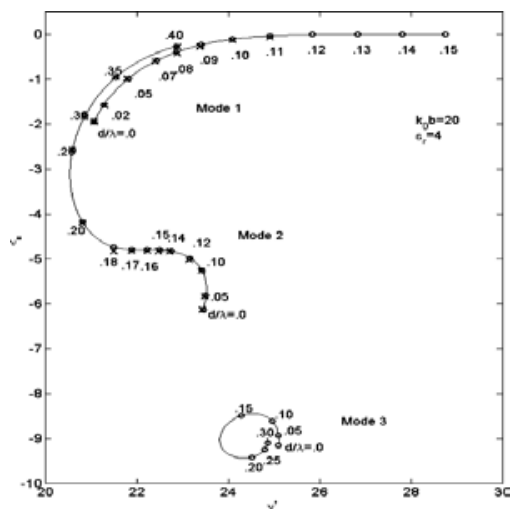

Fig. 3. TE incidence of 1-layer dielectric coated cylinder with $k_{0} b=20$ and $\epsilon_{r}=4$, where $b$ is the outer radius of the structure. Our results (circles) are shown with varying thicknesses of the dielectric coating. The results from ${ }^{6}$ are shown as X's.

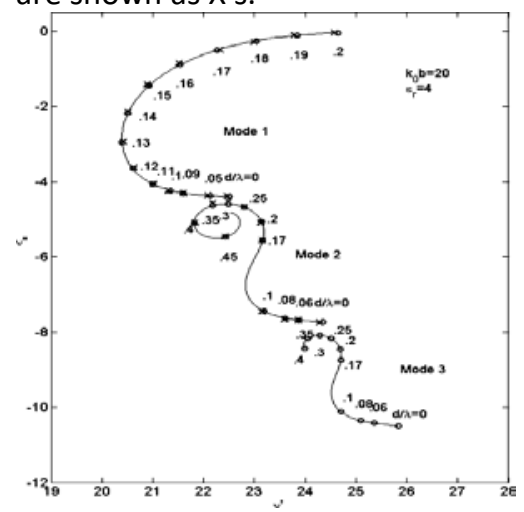

Fig. 4. TM incidence of 1-layer dielectric coated cylinder with $k_{0} b=20$ and $\epsilon_{r}=4$, where $b$ is the outer radius of the structure. Our results (circles) shown with varying thicknesses of the dielectric coating. The results from ${ }^{6}$ are shown X's.

Later, Sun et al. expanded previous works by implementing a lossy magnetic coating impinged by TE incidence. ${ }^{18}$ We duplicate the data presented in Fig. 9 of ${ }^{18}$ as seen in Fig. 5 . We note the $e^{-i \omega t}$ convention was used in. ${ }^{6,18}$ We've changed sign convention to $e^{j \omega t}$ in our comparisons. In all cases we see excellent agreement with our formulation, validating (20).

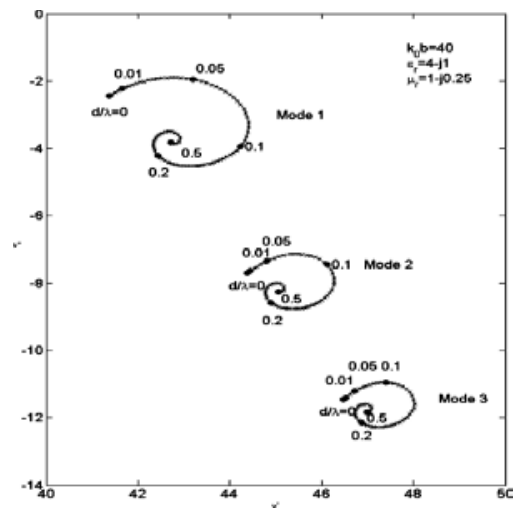

Fig. 5. TE incidence of 1-layer coated cylinder with $k_{0} b=40$ and $\epsilon_{r}=4-j 1, \mu_{r}=1-j 0.25$. These results show the azimuthal propagation constants with varying thickness of the coating. The solid line with dots is our data while the $x$ 's is the data extrapolated from. ${ }^{18}$ 


\section{B. Double Layer Case}

Next we duplicate the results from ${ }^{12}$ which used a double layer geometry. Our results duplicate those published for the single layer as seen in Fig. 6. However, there is some discrepancy when the second layer is added which is due to the Debye/Watson asymptotic approximations used in. ${ }^{12}$

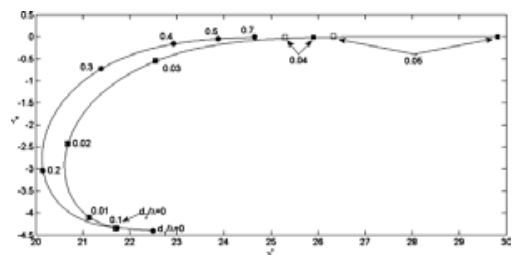

Fig. 6. TM incidence of 2 layer coated cylinder. Round dots track the single layer case of varying coating thicknesses with $\rho_{1}=3.1831 \lambda$ and $\epsilon_{r}=2.1$. The square dots track the change poles associated with the change in the second layer while the first layer is fixed at a thickness of . $1 \lambda$. The associated values of the 2-layer case are $a=3.0831 \lambda$, $\rho_{1}=3.831 \lambda, \epsilon_{r 1}=2.1, \epsilon_{r 2}=10$. Our results are shown as solid dots and the results from ${ }^{12}$ are shown as empty dots.

The Debye and Watson approximations are not valid everywhere and the relationship between the order and the argument dictates which expression can be used. Specifically, when $\left|v-k_{i} \rho_{i}\right| \geq|v|^{1 / 3}$ (where the product $k_{i} \rho_{i}$ makes up the Bessel function argument) the Debye approximations are used and otherwise the Watson approximations are used. ${ }^{23}$ It has been shown that this delimiter is not optimal and can introduce error into the calculation. ${ }^{21}$

Consider the case when the second layer thickness is . 04 $\lambda$. At this thickness, the differences in our results and those $\mathrm{in}^{12}$ are graphically noticeable. It can also be shown that at this thickness, the argument of the Bessel functions in $P, Q$ is close to the delimiter between using the Debye and Watson approximations. The Bessel function argument in $P, Q$ is made of $k_{1}=9.105$ and $\rho_{1}=3.183 \lambda$ and the associated order is $v=25.91-j .04$. Therefore, the delimiter expressions, $\left|v-k_{1} \rho_{1}\right|=3.07$ and $|v|^{1 / 3}=2.96$ are close and the accuracy of the asymptotic evaluation of the Bessel functions is questionable.

Also, it is important to note that the discrepancy is more apparent in the double layer equation (21) than in the single layer equation $(20)$ because the double layer equation contains more instances of the $P, Q$ expressions than does the single layer equation. This illustrates the effect of compounded error in stratified media.

Later in 2007, Sun et al. retrieved the azimuthal propagation constants of a 2-layered PEC cylinder with lossy material. ${ }^{2}$ In contrast to, ${ }^{12}$ the work in ${ }^{2}$ used the more accurate Olver asymptotic expressions for the Bessel functions. In Fig. 7 we duplicate Fig. 5 from. ${ }^{2}$ Our data tracks much better with these published results due to the use of the Olver asymptotic expressions used in. ${ }^{2}$

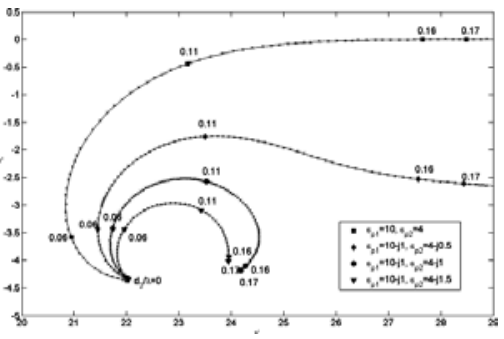


Fig. 7. TM incidence of 2-layer coated cylinder where $a=3.1331 \lambda, d_{1}=.05 \lambda$ and $d_{2}$ is varied from 0 to $.17 \lambda$. The solid lines with dots is our data while the $x^{\prime}$ s signify the data extrapolated from. ${ }^{2}$

\section{Multilayer Case}

In our final validation case, we duplicate Paknys and Wang's single layer case ${ }^{6}$ but divide the single layer into five equally spaced layers, all with the same material parameters as the single layer case. This allows us to validate the multilayer case and determine what type of error we can expect from the cascaded boundary conditions. Again, this approach is motivated by a lack of comparable multilayer cases in the published literature. The results are identical within graphical accuracy so we will not duplicate Figs. 3 or 4 . An error analysis comparing the results of the multilayer case and the single layer case showed the largest error to be $8 \times 10^{-6}$ for both real and imaginary parts of either TM or TE incidence.

\section{A Graded Index Multilayer Example}

We now turn to the first of two examples where our methodology allows a systematic variation of a multilayer structure. In each case, we provide a brief analysis demonstrating the physics of a multilayer structure. The focus is always on the analysis benefit of our method rather than on the particular physics involved. That being said, the observed phonomena are of interest in their own right.

$\mathrm{In}^{12}$ it was shown that by adding a second layer with a larger material parameter than the first layer, the attenuation constant of the azimuthal wave could be significantly reduced. We take this concept one step further to demonstrate the capabilities of our approach. We implement a material parameter gradient to reduce the attenuation constant further. We replace the first layer of the double layer example in Section IV-B with six equally spaced layers so that the cumulative thickness of the six layers is the same as the first layer of the double layer case. This replaces the single valued layer with a layer of graded material parameters that is approximated by six layers. This new first layer has a thickness of $.1 \lambda$ and the material parameters of the six layers within are $\mu_{r}=1$ and $\epsilon_{r}=2.1,5,6,7,8,9$ in order of innermost to outer. Then we vary the thickness of the last layer from 0 to $.03 \lambda$ which has material parameters of $\mu_{r}=1$ and $\epsilon_{r}=10$. The results can be seen in Fig. 8.

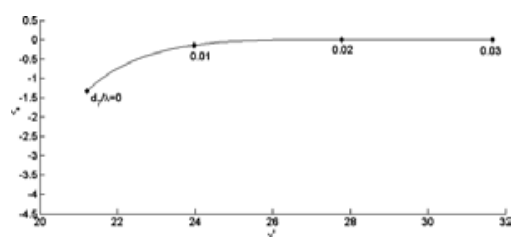

Fig. 8. Azimuthal propagation constants for a 7-layer dielectric coated cylinder. The thicknesses of the first 6 layers are constant at . $0167 \lambda$ and last layer thickness is varied from 0 to $.03 \lambda$. The cylinder radius is $a=3.0831 \lambda$.

The first data point in Fig. 8 corresponds with the one layer of graded material that is approximated by six layers without the additional last layer. This graded material with a total thickness of $0.1 \lambda$ has a smaller attenuation coefficient than the double layer example in Section IV-B with a total of thickness $0.22 \lambda$. These results show that a material parameter gradient can decrease the attenuation of the azimuthal wave more than with the addition of the second layer alone. More importantly, this example shows how this method can provide additional insight into the fields within a multilayered structure. 


\section{E. A Lossy Multilayer Example}

As a final example of the use of our methodology, we turn to Sun's paper ${ }^{2}$ investigating the effect of loss in the 2-layer dielectric coated cylinder. Our baseline geometry defines the PEC radius to be $\left(a_{1} / \lambda\right)=$ 3.0831 as in Sun's paper. The substrate layer is divided into four layers of $0.025 \lambda$ each summing up to the $0.1 \lambda$ thickness used by Sun. Finally, the outer superstrate layer thickness is varied from 0 to $0.04 \lambda$ to generate the root locus curves in the following figures.

Our lossless baseline geometry consists of a substrate formed from thin alternating low-high dielectric constant layers capped by a high dielectric constant superstrate layer. The specific dielectric profile from the inner to the outer layer is $\epsilon_{r}=[2.1,6.0,2.1,6.0,10.0]$. This baseline structure is included in each of the following figures for reference and can be compared with Fig. 2 of. ${ }^{2}$ The dielectric stack of alternating substrate layers has an effective dielectric constant higher than $\epsilon_{r 1}=2.1$ found in Sun's paper. The effect is to allow low attenuation trapped mode propagation to occur for slightly thinner superstrate layer thicknesses compared to Sun's baseline structure.

In Fig. 9, we examine the impact of introducing loss into one of the substrate layers. The inclusion of loss in any of the substrate layers has the general effect of creating a minimum attenuation value that can not be reduced by increasing the superstrate layer thickness. This is similar to the effect observed by Sun. In addition, we observe that as the loss is introduced into the layers progressively closer to the superstrate layer, the saturate attenuation level monotonically increases. This occurs despite the alternating nature of the dielectric constant stack. Finally, we note from Fig. 9 that the introduction of loss in the superstrate layer eliminates the saturate attenuation level, causing both the wave attenuation and phase constant to increase as function of layer thickness characteristic of a leaky wave mode.

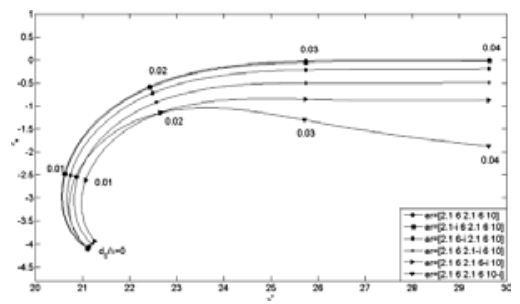

Fig. 9. Dominate mode propagation constant as a function of the superstrate thickness with the substrate multilayer structure consisting of $0.025 \lambda$ thick layers with the dielectric profiles given in the figure legend. The lossy layer creates an increasing saturation attenuation as a function of the proximity to the superstrate layer.

Fig. 10 illustrates the effect of introducing loss into both a substrate layer and the superstrate layer. As Sun observed, the dielectric loss of the superstrate will lead to a high attenuation mode as the thickness of the superstrate increases; with increased loss as the dielectric loss is increased. However we also observe another interesting effect as a result of introducing loss into the substrate layers progressively closer to the superstrate layer. Unlike the monotonic behavior seen in Fig. 9, here we see the value of the dielectric constant having an influence on magnitude of the attenuation. Specifically, the attenuation seen in the $\epsilon_{r}=[2.1,6.0-i, 2.1,6.0,10.0-i]$ structure is much greater than that observed in the $\epsilon_{r}=[2.1,6.0,2.1-i, 6.0,10.0-i]$ structure despite the fact the lossy layer is $0.025 \lambda$ closer to the superstrate. 


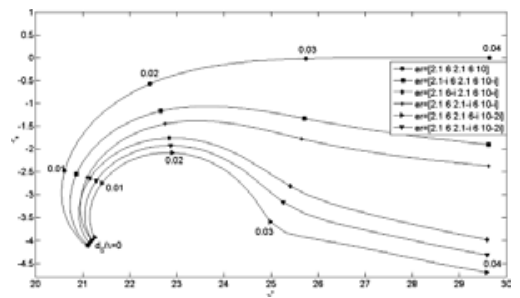

Fig. 10. Dominate mode propagation constant as a function of the superstrate thickness with the substrate multilayer structure consisting of $0.025 \lambda$ thick layers with the dielectric profiles given in the figure legend. The lossy superstrate layer doesn't allow trapped mode propagation.

Finally, in Fig. 11, we increase the loss in the superstrate layer beyond the threshold value where the root locus curls back on itself as the superstrate thickness is increased. This phenomena was also observed by Sun. Comparing Figs. 10 with 11, we see the larger superstrate loss dominates the more subtle effect of the dielectric constant returning to a monotonic relationship between the lossy substrate layer proximity to the superstrate layer.

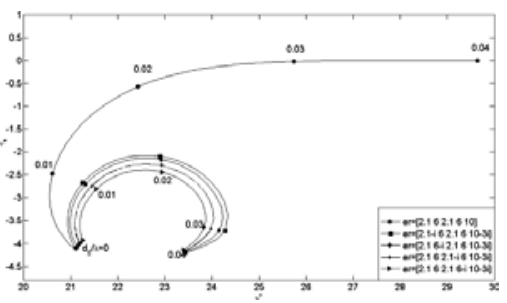

Fig. 11. Dominate mode propagation constant as a function of the superstrate thickness with the substrate multilayer structure consisting of $0.025 \lambda$ thick layers with the dielectric profiles given in the figure legend. As Sun observed in, ${ }^{2}$ when the superstrate loss exceeds a threshold value the root locus curls back on itself.

\section{SECTION V. Conclusion}

In this article we presented a Green's function approach to finding the azimuthal wave propagation constants for an n-layered dielectric coated PEC cylinder. Instead of the traditional asymptotic expressions for Bessel functions, we implemented a new numerical method from ${ }^{17}$ with better accuracy. The results were then validated with previously published results. This method is a general approach and can be applied to any stratification profile without additional analysis. We provided two brief examples to illustrate the benefits of this method when examining variations in a stratification profile. This approach lends itself to approximating the graded material parameters that are encountered in structures designed using transformational optics and metamaterials.

\section{ACKNOWLEDGMENT}

The authors would like to thank the reviewers for their insightful comments and suggestions. Their input lead to several interesting lines of inquiry. We believe the resulting manuscript is a stronger technical contribution to the community due in no small part to their excellent recommendations.

\section{References}

1. J. McGuirk, P. Collins, M. Havrilla, A. Wood, "A green's function approach to calculate scattering width for cylindrical cloaks", ACES, vol. 1, no. 1, pp. 1-1, 2009. 
2. J. Sun, L.-W. Li, "Dispersion of waves over a PEC cylinder coated with two-layer lossy dielectric materials", IEEE Trans. Antennas Propag., vol. 55, no. 3, pp. 877-881, Mar. 2007.

3. R. S. Elliot, "Azimuthal surface waves on circular cylinders", J. Appl. Phys., vol. 26, no. 4, pp. 368-376, 1955.

4. J. Wait, "On the excitation of electromagnetic surface waves on a curved surface", IEEE Trans. Antennas Propag., vol. 8, pp. 445-448, 1960.

5. K. Naishadham, H.-W. Yao, "An efficient computation of transient scattering by a perfectly conducting cylinder", IEEE Trans. Antennas Propag., vol. 41, no. 1, pp. 1509-1515, Nov. 1993.

6. R. Paknys, N. Wang, "Creeping wave propagation constants and modal impedance for a dielectric coated cylinder", IEEE Trans. Antennas Propag., vol. 34, no. 5, pp. 674-680, Dec. 1986.

7. R. Paknys, N. Wang, "Excitation of creeping waves on a circular cylinder with a thick dielectric coating", IEEE Trans. Antennas Propag., vol. 35, no. 12, pp. 1487-1489, Dec. 1987.

8. R. Paknys, D. R. Jackson, "The relation between creeping waves leaky waves and surface waves", IEEE Trans. Antennas Propag., vol. 53, no. 3, pp. 898-907, Mar. 2005.

9. N. Wang, "Electromagnetic scattering from a dielectric coated circular cylinder", IEEE Trans. Antennas Propag., vol. 33, no. 9, pp. 960-963, Sep. 1985.

10. A. Krasnojen, "Features of creeping waves propagation on the dielectriccoated circular cylinder", IEE Proc. Microw. Antenna Propagation, vol. 145, no. 2, pp. 179-183, Apr. 1998.

11. N. Albertsen, "Creeping wave modes for a dielectric coated cylinder", IEEE Trans. Antennas Propag., vol. 37, no. 12, pp. 1642-1644, Dec. 1989.

12. K. Naishadham, L. Felsen, "Dispersion of waves guided along a cylindrical substrate-superstrate layered medium", IEEE Trans. Antennas Propag., vol. 41, no. 3, pp. 304-313, Mar. 1993.

13. L. W. Pearson, "A ray representation of surface diffraction by a multilayer cylinder", IEEE Trans. Antennas Propag., vol. 35, no. 6, pp. 698-707, Jun. 1987.

14. A. Ward, J. Pendry, "Refraction and geometry in Maxwell's equations", J. Mod. Opt., vol. 43, no. 4, pp. 773-793, 1996.

15. D. Schurig, J. J. Mock, B. J. Justice, S. A. Cummer, J. B. Pendry, A. F. Starr, D. R. Smith, "Metamaterial electromagnetic cloak at microwave frequencies", Science, vol. 314, no. 5801, pp. 977-980, 2006.

16. H. E. Bussey, J. H. Richmond, "Scattering by a lossy dielectric circular cylindrical multilayer numerical values", IEEE Trans. Antennas Propag., pp. 723-725, 1975.

17. M. Kodama, "Algorithm 912: A module for calculating cylindrical functions of complex order and complex argument", ACM Trans. Math. Softw., vol. 37, no. 4, Feb. 2011.

18. J. Sun, C.-F. Wang, L. W. Li, M.-S. Leong, "Creeping waves along a perfectly conducting cylinder with a lossy magnetic coating", IEEE Trans. Antennas Propag. Lett., vol. 2, 2003.

19. J. McGuirk, Electromagnetic Field Control and Optimization Using Metamaterials, OH, WrightPatterson AFB:Air Force Institute of Technology(AU), 2009.

20. W. C. Chew, Waves and Fields in Inhomogeneous Media, New York:Van Nostran Reinhold, 1990.

21. R. Paknys, "Evaluation of hankel functions with complex argument and complex order", IEEE Trans. Antennas Propag., vol. 40, no. 5, pp. 569-578, May 1992.

22. N. Marcuvitz, "On field representations in terms of leaky modes and eigenmodes", IRE Trans. Antennas Propag., vol. AP-4, pp. 192-194, 1956.

23. L. B. Felsen, N. Marcuvitz, Radiation and Scattering of Waves, USA, NJ, Englewood Cliffs:Prentice Hall, 1973. 